Organo- and

Biocatalysis

\title{
Catalytic Enantioselective Oxaziridination
}

Key words

oxaziridines

cinchona alkaloids

aldimines

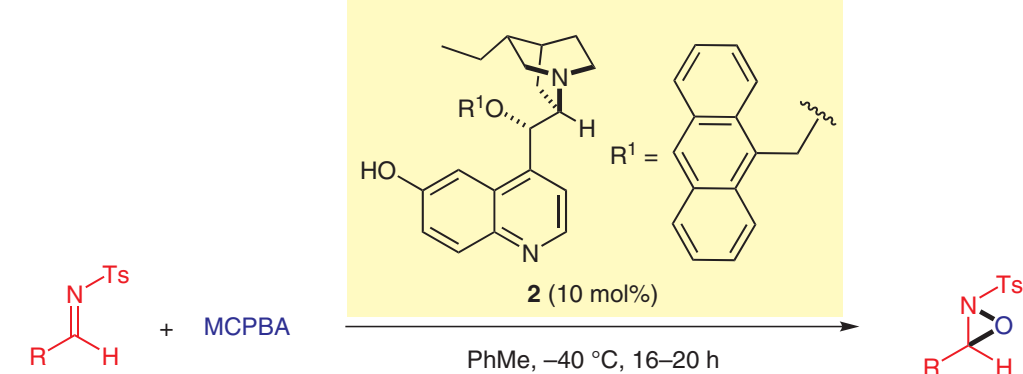

$\mathrm{R}=\mathrm{Ar}, \mathrm{Alk}$

1

Proposed mechanism:

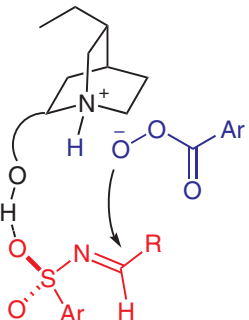

$\mathrm{R}=\operatorname{Ar}$ 50-97\% yield, er $=83: 17-97$

$\mathrm{R}=$ Alk 52-95\% yield, er = 73:27-97 only trans diastereomer

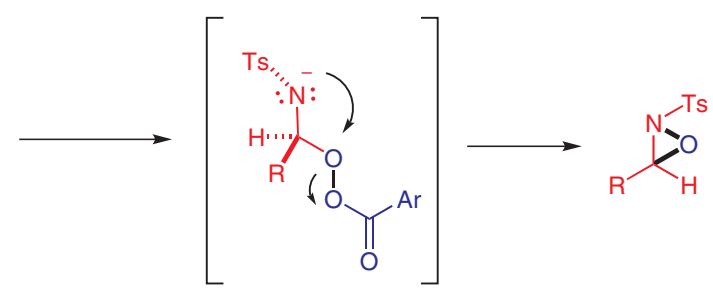

Significance: Jørgensen and colleagues disclose a catalytic asymmetric synthesis of oxaziridines $\mathbf{3}$ via the oxidation of aldimines $\mathbf{1}$ with MCPBA. Cinchona alkaloid derivative $\mathbf{2}$, which features a hydrogen-bonding site (phenolic group) and a Brønsted basic moiety (quinuclidine nitrogen) proved to be the optimal catalyst. A variety of electron-neutral and electron-poor aryl aldimines as well as alkyl aldimines underwent the oxaziridination with good to excellent yields and enantioselectivities. Kinetic studies indicated a pseudofirst order for both MCPBA and the aldimine, supporting the catalyst-substrate assembly model shown above. Competition experiments further indicated a stepwise mechanism.
Comment: Following the recent report of Antilla and co-workers on the hydroperoxidation of acyl imines with a Brønsted acid catalyst (Angew. Chem. Int. Ed. 2010, 49, 6589), the group of Jørgensen developed the first catalytic asymmetric aziridination protocol for various aldimines. Key to this transformation was the use of the bifunctional cinchona-derived catalyst 2 . Since the electronic nature of the $\mathrm{R}$ group in the aldimines (see scheme) did not affect the enantioselectivity, the authors proposed activation of the aldimine component via its tosyl group. The basic quinuclidine moiety was proposed to activate MCPBA by deprotonation. Since quinuclidine is known to undergo facile $\mathrm{N}$-oxidation by MCBPA under similar reaction conditions, it would be intriguing to examine if the real catalytic species is indeed oxidized at the tertiary nitrogen, by reisolating and characterizing the catalyst at the end of the reaction. 